\title{
Importancia del ultrasonido a la cabecera del paciente en el manejo urgente de la disnea
}

\author{
Joana Fontes ${ }^{1}$, Tiago Mendes, Raquel Costa, Joana Faria-Silva, Bárbara Sousa \\ 'Servicio de Medicina Interna, Hospital de Santa Luzia - Unidade Local de Saúde do Alto Minho, Viana do Castelo, Portugal
}

Recibido: 06/08/2021

Aceptado: 09/10/2021

En línea: 31/12/2021

Citar como: Fontes J, Mendes T, Costa R, Faria-Silva J, Sousa B. Importancia del ultrasonido a la cabecera del paciente en el manejo urgente de la disnea. Rev Esp Casos Clin Med Intern (RECCMI). 2021 (dic); 6(3): 15-17. doi: 10.32818/reccmi.a6n3a6.

Cite this as: Fontes J, Mendes T, Costa R, Faria-Silva J, Sousa B. The importance of point-of-care ultrasonography in the emergency management of dyspnea. Rev Esp Casos Clin Med Intern (RECCMI). 2021 (Dec); 6(3): 15-17. doi: 10.32818/reccmi.a6n3a6.

Autor para correspondencia: Joana Fontes. joana.pm.fontes@gmail.com

\section{Palabras clave \\ $\checkmark$ Ultrasonido a la cabecera del paciente \\ $\checkmark$ Sala de emergencia \\ - Tromboembolismo pulmonar}

\begin{tabular}{l} 
Keywords \\
\hline$\quad$ Point-of-care \\
$\quad$ ultrasonography \\
$\triangleright$ Emergencyroom \\
$\triangleright \quad$ Pulmonary \\
$\quad$ thromboembolism \\
\hline
\end{tabular}

\begin{abstract}
Resumen
El ultrasonido a la cabecera del paciente es una herramienta esencial en la evaluación inicial del paciente crítico. Presentamos el caso de una mujer de 83 años que ingresó por un cuadro de alteración súbita del estado de consciencia. A su llegada estaba confusa, con hipoxemia, taquicardia y signos de mala perfusión periférica. El ultrasonido a la cabecera del paciente mostró dilatación del ventrículo derecho compatible con la fuerte sospecha clínica de tromboembolismo pulmonar.

Este caso demuestra la importancia de incluir el ultrasonido a la cabecera del paciente en la evaluación de pacientes críticos, pues permite reducir el tiempo para el diagnóstico y tratamiento, mejorando su pronóstico.

Abstract
Point-of-care ultrasonography is an important tool in the initial assessment of critical patients. In this report, we
describe the case of an 83-year-old woman admitted to the emergency room due to a sudden unclear change of
consciousness. She was confused, hypoxemic, tachycardic, and had signs of poor perfusion. The point-of-care ul-
trasonography showed dilatation of the right ventricle, raising a solid suspicion of pulmonary thromboembolism.
This case supports the importance of including the point-of-care ultrasonography in the assessment of critically ill
patients, as it reduces the time for diagnosis and treatment, improving the patient's prognosis.
\end{abstract}

\section{Puntos destacados}

- El ultrasonido a la cabecera del paciente complementa la evaluación de la historia clínica y la exploración física en los pacientes críticos.

- Esta herramienta permite orientar un diagnóstico precoz y apoyar con rapidez las decisiones terapéuticas.

\section{Introducción}

El ultrasonido a la cabecera del paciente (UCP; más conocido en inglés, PointOf-Care UltraSonography [POCUS]) ha emergido como una herramienta importante en la evaluación inicial del paciente crítico ${ }^{1-3}$. Cada vez más se considera un complemento al examen físico, incluyendo información que permite identificar rápidamente las situaciones potencialmente fatales ${ }^{4}$

En este artículo se presenta un caso clínico de una emergencia médica donde la realización del POCUS permitió orientar la investigación etiológica y proporcionar un tratamiento más rápido.

\section{Caso clínico}

Antecedentes y enfermedad actual

Paciente de 83 años, caucásica con antecedentes personales de hipertensión arterial, dislipidemia, osteoartritis y accidente vascular cerebral isquémico 5 meses antes sin secuelas.

Fue admitida en Urgencias por un cuadro de alteración súbita del estado de consciencia con pérdida de conocimiento e incontinencia de esfínteres; sin referir movimientos involuntarios. Se intentó establecer contacto con familiares/ cuidadores, sin éxito, para completar la información y caracterizar el cuadro.

\section{Exploración física}

La paciente se presentaba prostrada, con discurso confuso, Glasgow de 14 puntos, pero sin asimetrías motoras en el examen neurológico. Estaba taquipneica (FR 30 cpm), con una saturación periférica de $\mathrm{O}_{2}$ de $90 \%$ con oxigenoterapia suplementaria a 28\%, taquicárdica (FC 150 bpm) y con presión arterial 100/50 $\mathrm{mmHg}$. 
En la exploración presentaba signos de mala perfusión (vasoconstricción periférica con frialdad), con pulsos periféricos palpables pero filiformes, ingurgitación venosa yugular y sin edema periférico. En la auscultación pulmonar el murmullo vesicular estaba conservado. Auscultación cardíaca rítmica y taquicardia, sin soplos. Se encontraba apirética y con una glucemia capilar de 210 $\mathrm{mg} / \mathrm{dL}$.

\section{Pruebas complementarias}

De las pruebas complementarias realizadas cabe destacar: gasometría arterial con insuficiencia respiratoria hipoxémica con ratio $\mathrm{PaO}_{2} / \mathrm{FiO}_{2}$ de 196 y lactato de $3,1 \mathrm{mmol} / \mathrm{L}$; ECG con taquicardia sinusal con bloqueo completo de rama derecha de reciente aparición.

Se realizó una ecocardioscopia que constató dilatación e hipocinesia del ventrículo derecho (VD) y un ventrículo izquierdo (VI) con D-shape, colocando como primera hipótesis diagnóstica la presencia de tromboembolismo pulmonar (Figura 1).

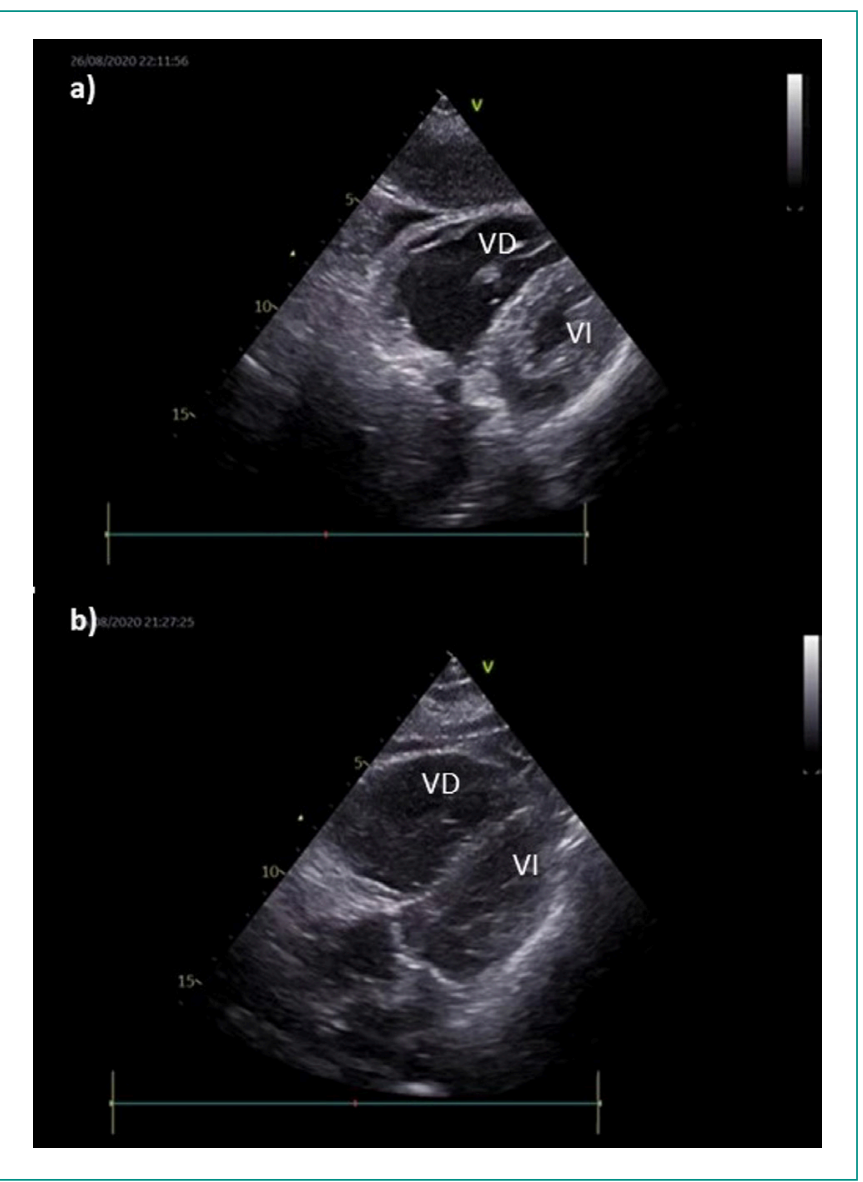

Figura 1. La evaluación ecográfica cardíaca en los planos paraesternal transversal (a) y subcostal de cuatro cavidades (b) evidencian la dilatación del VD. VI: ventrículo izquierdo; VD: ventrículo derecho.

La analítica reveló elevación de los valores séricos de los marcadores de necrosis miocárdica y del péptido natriurético tipo B (BNP) (Mioglobina $156 \mathrm{ng} / \mathrm{mL}$ [N 1-147 ng/mL], Troponina I 424,6 pg/mL [N <15,6 pg/mL], BNP 132,9pg/mL $[\mathrm{N}<100 \mathrm{pg} / \mathrm{mL}])$.

La angiografía de las arterias pulmonares por tomografía computarizada (TC) confirmó la presencia de tromboembolismo pulmonar (TEP) bilateral masivo y cardiomegalia, con predominio del ventrículo derecho (VD) (Figura 2).

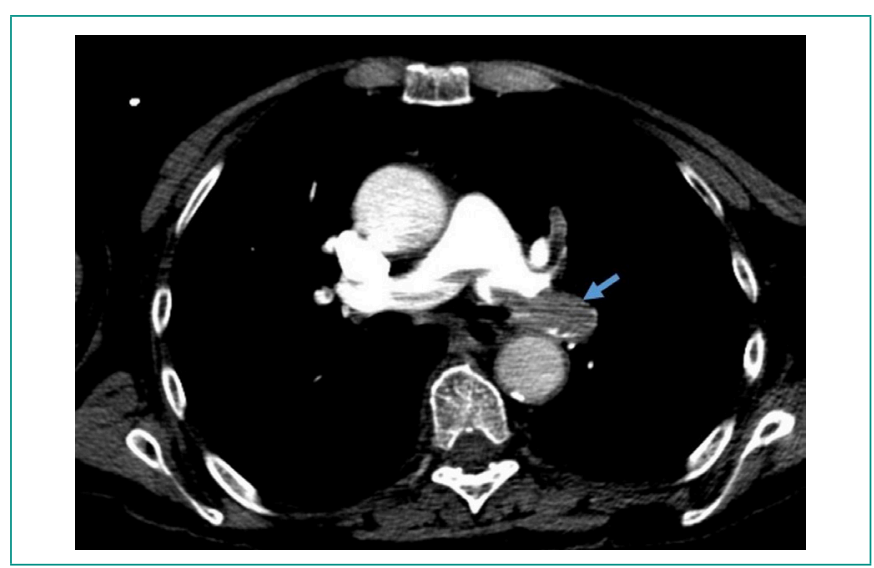

Figura 2. Angiografía de las arterias pulmonares porTC (plano axial). Defectos de perfusión de la arteria pulmonar compatibles con tromboembolismo pulmonar (flecha azul).

En Urgencias no se evaluó la presencia de trombosis venosa profunda de los miembros inferiores con ecografía clínica.

\section{Evolución}

Teniendo en cuenta las comorbilidades y la presentación clínica, se diagnostiCó un TEP de muy alto riesgo (Pulmonary Embolism Severity Index [PESI] clase V). El riesgo de mortalidad en estos pacientes a los 30 días es muy alto (10 a 24,5\%) por lo que se le sometió a tratamiento con trombólisis con tenecteplase, tras el cual presentó una mejoría significativa del perfil hemodinámico y la paciente ingresó en una Unidad de Cuidados Intermedios.

\section{Diagnóstico}

Tromboembolismo pulmonar de muy alto riesgo.

\section{Discusión}

El abordaje diagnóstico de la disnea aguda en Urgencias es un desafío. El POCUS se utiliza cada vez más en la evaluación de estos pacientes críticos, donde se ha de incluir el TEP en el diagnóstico diferencial ${ }^{5}$.

Muchos estudios han demostrado que, cuando se agrega el POCUS a la vía diagnóstica estándar, se consiguen diagnósticos más correctos, con significancia estadística ${ }^{6,7}$.

El POCUS posibilita una evaluación cualitativa de la anatomía y la función cardíacas en el paciente en fallo hemodinámico: permite observar la dilatación de las cavidades cardíacas; realizar una estimativa visual de la fracción de eyección del VI; detectar la presencia de derrame pericárdico, y estimar el diámetro de la vena cava inferior y su variabilidad durante el ciclo respiratorio, ayudando en la evaluación de la volemia del paciente'

La importancia del ultrasonido se reconoce en las directrices sobre el TEP de la European Society of Cardiology del año 2019, donde la presencia de signos inequívocos de sobrecarga de presión del VD pueden justificar la realización fibrinólisis urgente en pacientes en fallo hemodinámico si la angiografía por TC no está disponible 8 .

En esto caso clínico, la objetivación de una dilatación e hipocinesia del VD colocó el TEP como hipótesis diagnóstica más probable. Permitió direccionar las pruebas complementarias, diagnosticar y tratar con mayor brevedad proporcionando una rápida estabilización hemodinámica de la paciente. 


\section{Conclusiones}

El caso clínico descrito demuestra la importancia de integrar el POCUS en la evaluación del paciente crítico. Esto permite disminuir el tempo hasta el diag nóstico y posibilitar un tratamiento más adecuado.

No obstante, se necesita más investigación clínica que fortalezca las ventajas del POCUS y que confirme su impacto en el tiempo de hospitalización y en la reducción de la morbimortalidad.

\section{Bibliografía}

1. Moore CL, Copel JA. Point-of-care ultrasonography. N Engl J Med. 2011 364(8): 749-57. doi: 10.1056/NEJMra0909487.

2. Whitson MR, Mayo PH. Ultrasonography in the emergency department. Crit Care. 2016; 20(1): 227. doi: 10.1186/s13054-016-1399-x.

3. Chenkin J, Atzema CL. Contemporary Application of Point-of-Care Echocardiography in the Emergency Department. Can J Cardiol. 2018; 34(2): 109116. doi: 10.1016/j.cjca.2017.08.018.

4. Narula J, Chandrashekhar Y, Braunwald E. Time to Add a Fifth Pillar to Bedside Physical Examination: Inspection, Palpation, Percussion, Auscultation, and Insonation. JAMA Cardiol. 2018; 3(4): 346-350. doi: 10.1001/jamacardio.2018.0001

5. Daley Jl, Dwyer KH, Grunwald Z, Shaw DL, Stone MB, Schick A et al. Increased Sensitivity of Focused Cardiac Ultrasound for Pulmonary Embolism in Emergency Department Patients With Abnormal Vital Signs. Acad Emerg Med. 2019; 26(11): 1211-1220. doi: 10.1111/acem.13774.

6. Qaseem A, Etxeandia-Ikobaltzeta I, Mustafa RA, Kansagara D, Fitterman N, Wilt TJ; Clinical Guidelines Committee of the American College of Physicians. Appropriate Use of Point-of-Care Ultrasonography in Patients With Acute Dyspnea in Emergency Department or Inpatient Settings: A Clinical Guideline From the American College of Physicians. Ann Intern Med. 2021; 174(7): 985-993. doi: 10.7326/M20-7844.

7. Gartlehner G, Wagner G, Affengruber L, Chapman A, Dobrescu A, Klerings I et al. Point-of-Care Ultrasonography in Patients With Acute Dyspnea: An Evidence Report for a Clinical Practice Guideline by the American College of Physicians. Ann Intern Med. 2021; 174(7): 967-976. doi: 10.7326/M205504.

8. Konstantinides SV, Meyer G, Becattini C, Bueno H, Geersing GJ, Harjola VP et al.; ESC Scientific Document Group. 2019 ESC Guidelines for the diagnosis and management of acute pulmonary embolism developed in collaboration with the European Respiratory Society (ERS). Eur Heart J. 2020; 41(4): 543-603. doi: 10.1093/eurheartj/ehz405. 